BIHEP-TH-97-50

\title{
H-DIHYPERON IN QUARK CLUSTER MODEL
}

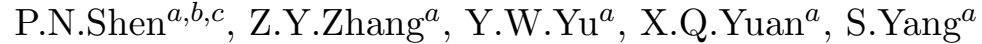

\author{
a. Institute of High Energy Physics, Chinese Academy of Sciences, P.O.Box 918(4), \\ Beijing 100039, China \\ b. China Center of Advanced Science and Technology (World Laboratory), \\ P.O.Box 8730, Beijing 100080, China \\ c. Institute of Theoretical Physics, Chinese Academy of Sciences, P.O.Box 2735, \\ Beijing 100080, China
}

\begin{abstract}
The $\mathrm{H}$ dihyperon $(\mathrm{DH})$ is studied in the framework of the $\mathrm{SU}(3)$ chiral quark model. It is shown that except the $\sigma$ chiral field, the overall effect of the other $\mathrm{SU}(3)$ chiral fields is destructive in forming a stable $\mathrm{DH}$. The resultant mass of $\mathrm{DH}$ in a three coupled channel calculation is ranged from $2225 \mathrm{MeV}$ to $2234 \mathrm{MeV}$.
\end{abstract}

${ }^{1}$ This work was partly supported by the National Natural Science Foundation of China 
In 1977, Jaffe predicted DH [1], a six-quark state with strangeness $(s)$ being -2 and $J^{P}=0^{+}(S=0, T=0)$, by using a simple color magnetic interaction in the MIT bag model. Since then, many theoretical [2, 4, 3, 5, 6, 7] and experimental [8] (and references therein) efforts have been devoted to the DH study. There were so many theoretical predictions of $\mathrm{DH}$ which are quite different in different models. For instance, by using the MIT bag model, Jaffe gave a binding energy of about $80 \mathrm{MeV}$ below the two $\Lambda$ threshold $\left(E_{\Lambda \Lambda}\right)$ [1; in terms of the Skyrme model, Balachandran et al. even showed a larger binding energy of about several hundred $\mathrm{MeV}$ [2]; by employing the cluster model, Yazaki et al. predicted the energy of DH from about $10 \mathrm{MeV}$ above $E_{\Lambda \Lambda}$ to about $10 \mathrm{MeV}$ below $E_{\Lambda \Lambda}$ [4, 14]; also, by using the cluster model but different interaction with Yazaki's, Straub et al. announced a binding energy around $20 \mathrm{MeV}$ [3]; in terms of Quantum Chromodynamics $(Q C D)$ sum rules, Kodama et al. gave a binding energy around $40 \mathrm{MeV}$ although the error bar was quite large; considering mutually the two-cluster and six-quark cluster configurations, Wolfe et al. obtained a deeply bound $\mathrm{DH}$ with a binding energy about several hundred $\mathrm{MeV}$ [6]; and by employing a quark model without the one-gluon-exchange interaction (OGE), Glozman et al. announced the non-existence of a bound $\mathrm{DH}[\mathrm{]}]$. On the other hand, there is no experimental evidence showing the existence of DH up to now. The provided lower limit of the DH mass is about $2200 \mathrm{MeV}$, 15].

The reason for carrying out such researches is straightforward. According to the feature of the color magnetic interaction (CMI) in the one-gluon-exchange potential (OGE), when the strangeness of the system concerned is equal to $-2, \mathrm{~S}=0$ and $(\lambda \mu)_{f}=(00)$, the expectation value of CMI presents more attractive feature than those contributed by two $\Lambda$ baryons. As a consequence, six quarks could be squeezed in a small region, the typical short-range $Q C D$ behavior would be demonstrated and some new physics might be revealed. Therefore, studying DH will be rather significant in understanding the quark characteristics of the wave function of the multiquark system and the short-range behavior of the $Q C D$ theory.

On the other hand, till now, there still exist some uncertainties in the nucleon-hyperon $(N Y)$ interaction on the baryon-meson degrees of freedom, especially in the short-range part, so as the hyperon-hyperon $(Y Y)$ interaction and the prediction of $\mathrm{DH}$. Therefore, the next generation, the quark-gluon degrees of freedom, may be a more effective base to establish the relations among the nucleon-nucleon $(N N), N Y$ and $Y Y$ interactions, and consequently to give a more reliable prediction of $\mathrm{DH}$. As well known, most nuclear phenomena are just of the low energy approximation of $Q C D$. There exist lots of nonperturbative $Q C D(N P Q C D)$ effects. Unfortunately, nowadays one still cannot solve NPQCD directly, and has to employs certain $Q C D$ inspired models. The $S U(3)$ chiral quark model is just one of the most successful 
ones. In that model, the couplings between chiral fields and quark fields were introduced to describe the short- and medium-range $N P Q C D$ effects, and a more reasonable quark-quark interaction $V_{q-q} \quad(q=u, d$, and $s)$ [1] was obtained. With that $V_{q-q}$, one could mutually describe the experimental $N N$ scattering phase shifts, available $Y N$ scattering cross sections 11] and some properties of single baryons including the empirical masses of single baryon ground states 12, 13]. Extrapolating that model to the $s=-2$ system, one can study the $Y Y$ interaction, double strangeness hypernuclei, DH and etc.. In this letter, we would choose $\mathrm{DH}$ as a target, because it is a simplest case with two strangeness and its structure is relative simple so that the interaction can be preliminarily examined by the present experimental finding although it is only a lower limit of the DH mass, and the short-range behavior can be revealed.

It is clear that both the DH structure and $V_{q-q}$ would affect the theoretical prediction of the DH mass. Here, we first briefly introduce the employed interaction. The Hamiltonian of a six-quark system in the $\mathrm{SU}(3)$ chiral quark model reads

$$
H=T+\sum_{i<j}\left(V_{i j}^{C O N F}+V_{i j}^{O G E}+V_{i j}^{P S}+V_{i j}^{S}\right)
$$

where $T$ denotes the kinetic energy operator of the system and $V_{i j}^{C O N F}, V_{i j}^{O G E}, V_{i j}^{P S}$ and $V_{i j}^{S}$ represent the confinement, one-gluon exchange, pseudo-scalar chiral field induced and scalar chiral field induced potentials between the i-th and j-th quarks, respectively. The confinement potential is phenomenologically taken as

$$
V_{i j}^{C O N F}=-\left(\lambda_{i}^{a} \lambda_{j}^{a}\right)_{c}\left(a_{0} i j+a_{i j} r_{i j}^{2}\right)
$$

which describes the long range nonperturbative $Q C D$ effect. The introduced $a_{0}$ ij terms which take different values for different $q-q$ pairs are called zero-point energy terms. They guarantee that the empirical thresholds of considered channels can more accurately be reproduced. The short range perturbative OGE potential is chosen to be the commonly used form [9, 10, 11, 12]. In order to restore the important symmetry of strong interaction, the chiral symmetry, we introduce $\mathrm{SU}(3)$ chiral fields coupling to quark fields so that the medium-range $N P Q C D$ effects can be described [9, 10, 11]. The pseudoscalar-field-induced potentials are:

$$
V_{i j}^{P S}=C\left(g_{c h}, m_{\pi_{a}}, \Lambda\right) \frac{m_{\pi_{a}}^{2}}{12 m_{i} m_{j}} \cdot f_{1}\left(m_{\pi_{a}}, \Lambda, r_{i j}\right)\left(\overrightarrow{\sigma_{i}} \cdot \overrightarrow{\sigma_{j}}\right) \cdot\left(\lambda_{i}^{a} \lambda_{j}^{a}\right)_{f}
$$


and the scalar-field-induced potentials are:

$$
V_{i j}^{S}=-C\left(g_{c h}, m_{\sigma_{a}}, \Lambda\right) \cdot f_{2}\left(m_{\sigma_{a}}, \Lambda, r_{i j}\right) \cdot\left(\lambda_{i}^{a} \lambda_{j}^{a}\right)_{f}
$$

where the subscript $f$ denotes that the operators in parentheses are in flavor space. The expressions of $f_{i}, Y$, and $C$ are shown in Ref. 11. To retain the important chiral symmetry as much as possible, we take all chiral-quark coupling constants to be the same value

$$
\frac{g_{c h}^{2}}{4 \pi}=\frac{9}{25} \frac{g_{N N \pi}^{2}}{4 \pi}\left(\frac{m_{q}}{M_{N}}\right)^{2}
$$

In Eq.(3), $\pi_{a}$ with $(a=1,2, \ldots, 8$, and 0$)$ correspond to the pseudoscalar fields $\pi, K, \eta_{8}$ and $\eta_{0}$, respectively, and $\eta$ and $\eta^{\prime}$ are the linear combinations of $\eta_{0}$ and $\eta_{8}$ with the mixing angle $\theta$. In Eq.(4), $\sigma_{a}$ with $(a=1,2, \ldots, 8$, and 0$)$ correspond to the scalar fields $\sigma^{\prime}, \kappa, \epsilon$ and $\sigma$, respectively.

In solving this six-quark system problem, the first selected set of model parameters is that used in Ref. [11]. This is because that with this set of parameters, almost all empirical partial wave phase shifts of the $N-N$ scattering can be well re-produced, meanwhile the available cross sections of $N-Y$ processes can reasonably be explained, some masses of baryon ground states can accurately be obtained, and some properties of baryons such as EM transition rates and etc. can better be understood [13, 12]. Thus, the predicted mass of DH is based on a more solid ground, and the reliability of the prediction is increased. Furthermore, the possible range of the DH mass can be tested by shifting the values of model parameters within reasonable regions.

Then, we show how to choose the model space in solving the bound state problem of a six-quark system. There are two types of possible configurations in studying the structure of $\mathrm{DH}$.

\section{(1) Six-quark cluster configuration.}

In this configuration, the trial wave function can be expressed as the linear combination of differently sized basis functions:

$$
\Psi_{(\lambda \mu)_{f} T S}=\sum_{i} C_{i} \Phi_{(\lambda \mu)_{f} T S}\left(\omega_{i}\right)
$$

with the basis function

$$
\Phi_{(\lambda \mu)_{f} T S}\left(\omega_{i}\right)=\phi\left[(0 s)^{6}, \omega_{i}\right] \chi_{(\lambda \mu)_{f}}^{f \sigma} \chi_{(00)}^{c},
$$


where $\phi\left[(0 s)^{6}, \omega_{i}\right]$ is the orbital wave function $\left(\omega=\frac{1}{m b^{2}}\right)$, and $\chi_{(\lambda \mu)_{f}}^{f \sigma}$ and $\chi_{(00)}^{c}$ denote the wave functions in the flavor-spin and color spaces, respectively. This trial wave function is in the pure symmetry-basis-function space. In this configuration space, $(0 s)^{6}$ configurations which describe the breath mode are considered only.

\section{(2) Two-cluster configuration.}

In this configuration, there exist three possible channels: $\Lambda \Lambda, N \Xi$ and $\Sigma \Sigma$. In the framework of Resonating Group Method (RGM), the trial wave function of DH can be written as

$$
\Psi=\alpha|\Lambda \Lambda\rangle+\beta|N \Xi\rangle+\gamma|\Sigma \Sigma\rangle
$$

with the two-cluster wave function

$$
\left|B_{1} B_{2}\right\rangle=\mathcal{A}\left[\phi_{B_{1}} \phi_{B_{2}} \chi_{\text {rel }} \mathcal{R}_{c m}\right]_{S T=00}
$$

where $\mathcal{A}$ stands for the antisymmetrizer, $\phi_{B_{1}(2)}$ denotes the wave function of the cluster $B_{1(2)}, \chi_{\text {rel }}$ represents the trial wave function of the relative motion between clusters $B_{1}$ and $B_{2}$ and $\mathcal{R}_{c m}$ is the wave function of the total center of mass motion. This trial wave function is in the physics-basis-function space. The physical picture of this configuration is that in the compound region of two-interacting clusters (or composite particles), there might exist a bound state or a resonance.

Let us define a quantity

$$
E_{H}=M_{H}-M_{\Lambda \Lambda}
$$

where $M_{H}$ and $M_{\Lambda \Lambda}$ denote the mass of $\mathrm{DH}$ and two $\Lambda$ 's, respectively. Apparently, $E_{H}<0$ stands for a stable DH against weak decay. $E_{H}$ (or $M_{H}$ ) can be obtained by solving the Schrödinger equation in which the above mentioned potentials are employed. The results with different configurations are discussed in the following.

(1) Six-quark cluster case.

In this case, $\omega_{i}$ are taken as variational parameters. By using the variational method, one can minimize the Hamiltonian matrix element with respect to $\omega_{i}$. The resultant masses of DH are tabulated in Table I. 


\section{Table I. $\quad E_{H}(M e V)^{\dagger}$ in the six-quark cluster case}

\begin{tabular}{|c|c|}
\hline$V^{O G E}+V^{C O N F}$ & 311 \\
\hline$V^{O G E}+V^{C O N F}$ & 127 \\
$+V^{\pi}+V^{\sigma}$ & \\
\hline$V^{O G E}+V^{C O N F}$ & 276 \\
$+V^{P S}+V^{S}$ & \\
\hline \multicolumn{2}{|c|}{ Parameters used are those in ref. 11.1.}
\end{tabular}

It is shown that if one only employ OGE and confinement potentials, the mass of six-quark cluster is $311 \mathrm{MeV}$ heavier than those of two $\Lambda$ 's, $M_{\Lambda \Lambda}$. When one additionally employs $V^{\pi}$ and $V^{\sigma}$, the mass of $\mathrm{DH}$ would decrease, but it still $127 \mathrm{MeV}$ heavier than $M_{\Lambda \Lambda}$. However, when one includes all SU(3) chiral pseudoscalar and scalar fields, namely employs the other chiral fields in additional to the $\pi$ and $\sigma$ fields, the corresponding mass becomes larger again, which is $276 \mathrm{MeV}$ heavier than $M_{\Lambda \Lambda}$. This means that the couplings between $\sigma$ chiral field and quark fields cause additional attraction, which is helpful to reduce the mass of the sixquark system. On the contrary, the overall effect of the contributions from other $\mathrm{SU}(3)$ chiral fields provides a repulsive feature so that $\mathrm{DH}$ is quite hard to form. Moreover, no matter in which case, the mass of $\mathrm{DH}$ is heavier than $M_{\Lambda \Lambda}$, namely $\mathrm{DH}$ is not a stable particle against to strong decay to $\Lambda \Lambda$ and/or $N \Xi$. Therefore, a model space with $(0 s)^{6}$ six-quark cluster structure only may not be a favored model space in studying the DH structure.

\section{(2) Two-cluster case.}

In RGM, to solve the bound state problem, one usually expands the unknown relative wave function $\chi_{\text {rel }}$ by using locally peaked Gaussian basis functions

$$
\chi_{r e l}=\sum_{i} c_{i} \chi_{i}
$$

where $c_{i}$ 's are variational parameters [17].

Due to the Pauli principle, there exists a forbidden degree in the six-quark trial function 16

$$
\Psi^{\text {forbidden }}=\mathcal{A}\left(\frac{1}{\sqrt{3}}|\Lambda \Lambda\rangle+\frac{1}{\sqrt{3}}|N \Xi\rangle+|\Sigma \Sigma\rangle\right) .
$$

This forbidden degree and almost forbidden degrees can be detected by examining the zero and almost zero eigenvalues of the normalization kernel, respectively [18]. In particular, in the 
bound state RGM calculation, a component with the inter-cluster distance to be zero in the trial wave function, which is just a six-quark cluster configuration with the [6] symmetry, has to be included so that the behaviors of two clusters at the shorter inter-cluster distance can be well described and the stable and reliable solutions can be obtained. As a side-effect, the disturbances from the forbidden and almost forbidden degrees become serious. Sometimes, these disturbances would spoil the numerical calculation and produce non-physical results. Therefore, only after all the non-physical degrees are completely eliminated, the resultant energy of the bound state can be trusted. Due to the aforesaid reasons, at this moment, it may not be necessary to further carry out the mixing of the configurations (1) and (2). Moreover, in practice, eliminating the non-physical degree can be realized by performing the off-shell transformation. Then, carrying out the variational procedure, one can obtain the mass of $\mathrm{DH}$ or $E_{H}$. The resultant $E_{H}$ 's are tabulated in Table II.

Table II. $E_{H}(\mathrm{MeV})$ and $\mathcal{R}(f m)$ in the two-cluster case $\mathbf{f}^{\dagger}$.

$\left.\begin{array}{|c|c|c|c|c|}\hline \multicolumn{2}{|c|}{} & (|\Lambda \Lambda\rangle) & \left(\begin{array}{r}|\Lambda \Lambda\rangle \\ +|N \Xi\rangle\end{array}\right) & \left(\begin{array}{r}|\Lambda \Lambda\rangle \\ +|N \Xi\rangle \\ +|\Sigma \Sigma\rangle\end{array}\right)\end{array}\right)$

$\dagger$ Parameters used are those in ref. [1] and $\mathcal{R}$ denotes the root-mean-squared radius of DH.

It is shown that in the first $\left(V^{O G E}+V^{C O N F}\right)$ and third $\left(V^{O G E}+V^{C O N F}+V^{P S}+V^{S}\right)$ cases, the results do not support a bound DH. In the second case $\left(V^{O G E}+V^{C O N F}+V^{\pi}+V^{\sigma}\right)$, the 
one-channel calculation $(|\Lambda \Lambda\rangle)$ result $\left(E_{H}=5.47 \mathrm{MeV}\right)$ also does not support a bound state, but, the two- channel $(|\Lambda \Lambda\rangle+|N \Xi\rangle)$ and three-channel $(|\Lambda \Lambda\rangle+|N \Xi\rangle+|\Sigma \Sigma\rangle)$ calculations show a bound state with the binding energies of $38.71 \mathrm{MeV}$ and $65.80 \mathrm{MeV}$, respectively. These results can roughly be explained by the interaction matrix elements. The contribution from $O G E$ shows repulsive feature in the $\Lambda \Lambda$ channel and attractive features in both $N \Xi$ and $\Sigma \Sigma$ channels, and the net contribution from $\pi$ and $\sigma$ fields at the short distance demonstrates the more attractive character in the $N \Xi$ and $\Sigma \Sigma$ channels than that in the $\Lambda \Lambda$ channel. However, due to the existences of two strange quarks in the DH system, the chiral clouds with strangeness surrounding interacting baryons become important. Thus, in our opinion, all the $\mathrm{SU}(3)$ chiral fields should be considered. In fact, after including these fields, the aforesaid over-strong attraction disappears, and the results in all cases become smooth. This phenomenon can be understood by the matrix elements of the spin-flavor-color operators of chiral fields, namely $s f c$ coefficients, which show that the contributions from $\sigma$ and $\eta_{0}$ present the attractive character and those from the other mesons, i.e., $\pi, K, \eta_{8}, \sigma^{\prime}, \kappa$ and $\varepsilon$, are repulsive. Moreover, in the third case, although all coupled channel calculations do not show a bound $\mathrm{DH}$, in comparison with the result in the six-quark cluster case, the inclusion of additional channel would reduce the mass of DH. Eventually, the resultant mass of DH is around the $\Lambda \Lambda$ threshold.

To further understand the calculated $E_{H}$, we also list the corresponding root-meansquared radii $(\mathcal{R})$ of $D H$ in different cases in Table II. These numbers indicate that in the two- and three-channel calculations, if one considers $O G E, \pi$ and $\sigma$ only, the root-meansquared radii of $D H$ are $0.75 \mathrm{fm}$ and $0.72 \mathrm{fm}$ for the two and three coupled channel cases, respectively. Therefore, $D H$ is a bound state. In all the other cases, the resultant $\mathcal{R}$ values are greater than $1.4 \mathrm{fm}$, thus $D H$ is no longer bound.

How the values of major model parameters affect the resultant mass of DH is also studied. Three coupled channel results are used as samples to demonstrate these effects. When the width parameter $b$ increases, the corresponding $E_{H}$ value changes to a smaller value. Decreasing the mass of $\sigma$, would make the DH mass lighter. Moreover, a smaller $s$ quark mass $m_{s}$ corresponds to a lower DH mass, and decreasing the mixing angle $\theta$ would just lower the $\mathrm{DH}$ mass in a very small amount. Among these parameters, the mass of $\sigma$ would give the biggest effect on $E_{H}$.

Finally, we give the possible mass range of $\mathrm{DH}$. As mentioned above, the $N N$ scattering phase shifts and the $N Y$ scattering cross sections as well as the mass of DH depend on the 
values of model parameters. Our calculation showed that except the set of model parameters used in Ref.[11], another set of model parameters, say $b=0.53 \mathrm{fm}, m_{\sigma}=600 \mathrm{MeV}, m_{s}=$ $430 \mathrm{MeV}, \quad \theta=-23^{\circ}, \quad \Lambda_{P S, \sigma^{\prime}}=987 \mathrm{MeV}, \quad \Lambda_{\kappa, \epsilon, \sigma^{\prime}}=1381 \mathrm{MeV}$, which are almost the values in limits, can also fit the experimental $N N$ and $N Y$ data 19. With this set of model parameters, the resultant $E_{H}$ is $-6.9 \mathrm{MeV}$. Further extending these parameter values to their physical limits with which the empirical $N N$ and $N Y$ data cannot even been reproduced, one can obtain the upper and lower bounds of the DH mass. When $b=0.6 \mathrm{fm}, m_{\sigma}=550 \mathrm{MeV}$, the lower bound of $E_{H}$ is $-9.1 \mathrm{MeV}$. On the other direction, if one takes $b=0.48 \mathrm{fm}, m_{\sigma}=$ $675 \mathrm{MeV}$, the upper bound of $\mathrm{DH}$ is around $4.9 \mathrm{MeV}$.

From above calculations, one finds that in the framework of our SU(3) chiral-quark model, as long as one picks up a set of model parameters which satisfy the stability conditions, the masses of the ground states of baryons and meanwhile can be used to fit the experimental $N N$ and $N Y$ scattering data, the resultant mass of $\mathrm{DH}$ would be rather stable and would be ranged in a very small region. This mass is consistent with the present experimental finding and reflects that the $\mathrm{SU}(3)$ chiral-quark model is reasonable.

As a summery, one may have following conclusions. The six-quark system with strangeness being $-2, J^{P}=0^{+}(\mathrm{S}=0, \mathrm{~T}=0)$ is studied in two possible model spaces. One is in a six-quark cluster configuration space with breath mode, and the other is in a two-cluster configuration space with three possible channels. It is shown that the $(0 s)^{6}$ model space is not larger enough even the breath mode is considered. Therefore, the mass of DH in this model space is generally $100 \sim 300 \mathrm{MeV}$ heavier than that in the two-cluster model space. The similar result also appears in the other six-quark system calculation, say Deltaron $\left(d^{*}\right)$ [10]. In the two-cluster configuration case, the result shows that the mass of $\mathrm{DH}$ is ranged from $2225 \mathrm{MeV}$ to $2234 \mathrm{MeV}$ if the experimental $N N$ and $N Y$ data should simultaneously be reproduced. It seems that all the $\mathrm{SU}(3)$ chiral fields must be considered in studying $\mathrm{DH}$. In this case, the $\mathrm{SU}(3)$ chiral fields surround the baryons and make the baryons more stable and independent. Therefore, the interaction between two baryons becomes weaker so that it is hard to form a stable six-quark particle. It is also shown that the lower and upper bounds of the DH mass in the $\mathrm{SU}(3)$ chiral quark model are $2223 \mathrm{MeV}$ and $2237 \mathrm{MeV}$, respectively.

\section{Acknowledgement}

We would like to thank Prof. A.Faessler's fruitful discussion during the Symposium Symmetry and Dynamics in Nuclear and Low Energy Particle Physics in Blaubeuren-Tuebingen, Germany. 


\section{References}

[1] R.L.Jaffe, Phys. Rev. Lett. 38, 195 (1977).

[2] A.P.Balachandran, et al., Phys. Rev. Lett. 52, 887 (1984);

R.L.Jaffe,C.L.Korpa, Nucl. Phys. B 258, 468 (1985);

S.A.Yost, C.R.Nappi, Phys. Rev. C 32, 816 (1985).

[3] U.Straub, Z.Y.Zhang et al., Phys. Lett. B 200, 241 (1988).

[4] M.Oka, K.Shimizu, K.Yazaki, Phys. Lett. B 130, 365 (1983).

[5] N.Kodama, M.Oka, T.Hatsuda, Nucl. Phys. A 580, 445 (1994).

[6] C.E.Wolfe, K.Maltman, Phys. Lett. B 393, 274 (1997).

[7] L.Ya.Glozman, et al., nucl-th/9705011.

[8] K.Imai, Nucl. Phys. A 553, 667c (1993).

[9] Y.W.Yu, Z.Y.Zhang, P.N.Shen, L.R.Dai, Phys. Rev. C 52, 3393 (1995).

[10] X.Q.Yuan, Z.Y.Zhang, Y.W.Yu, P.N.Shen, High Ener.Phys.Nucl.Phys., (In press).

[11] Z.Y.Zhang, Y.W.Yu, P.N.Shen, L.R.Dai, A.Faessler, U.Straub, Nucl. Phys. A 625, 59 (1997).

[12] P.N.Shen, Y.B.Dong, Z.Y.Zhang, Y.W.Yu, T.-S.H.Lee, Phys. Rev. C 55, 2024 (1997).

[13] H.Chen, Z.Y.Zhang, High Ener.Phys.Nucl.Phys. 20, 937(1996).

[14] K.Yazaki, "Hadrons and Nuclei with Strangeness", Proceedings of the First Sino-Japan Symposium on Strangeness Physics, (to be published in 1998).

[15] K.Imai, "Hadrons and Nuclei with Strangeness", Proceedings of the First Sino-Japan Symposium on Strangeness Physics, (to be published in 1998).

[16] M.Oka, K.Shimizu, K.Yazaki, Nucl. Phys. A 464, 700 (1987).

[17] M.Kamimura, Prog.Theor.Phys. Suppl.62, 236(1977).

[18] K.Wildermuth, Y.C.Tang, "A Unified Theory of the Nucleus" (Academic Press, New York, 1977).

[19] S.Yang, P.N.Shen, Y.W.Yu, Z.Y.Zhang, Nucl. Phys. A 635, 146 (1998). 